\title{
PUBBLICAZIONI RECBNTI
}

A. Mannhem. - Construclion des Centres de courbure des lignes décrites dans le mouvement d'une figure qui glisse sur son plan (Extrait du Journal de I'École polytechnique XXXVII Cahier).

E. Catalan - Mémoire sur les surfaces dont les rayons de courbure en chaque point sont égaux, et des signes contraires (Extrait du Journal de l'École polytechnique XXXVII Cahier).

V. A. Lebesgue. - Exercices d'analyse numérique, extraits, commentaires, et recherches relatifs à l'analyse indeterminée. Paris 1859,1 . vol. in $8^{\circ}$.

Cossalı. - Scritti inediti pubblicati da Baldassarre Boncompagni, seguiti da un'Appendice contenente quattro Lettere dirette al medesimo P. Cossali, ed una Nota intorno a queste lettere. Roma, Tipografia delle Belle Arti, 1857. 1. Yol. in 4:

Leonardo Pisano - Scritti inediti pubblicati da Baldassarre Boncompagni. Volume I. (Leonardi Pisani - Liber Abbaci). Roma. Tipografia delle scienze matematiche e fisiche, 1857. 1. Yol. in 4\% 